\title{
Exact Boundary Controller Design for a Kind of Enhanced Oil Recovery Models
}

\author{
Ping Guo, ${ }^{1}$ Changhua Wei, ${ }^{2}$ Wenjun Xiong, ${ }^{3}$ and Chunlan Zhao ${ }^{3}$ \\ ${ }^{1}$ State Key Lab of Oil and Gas Reservoir Geology and Exploitation, Southwest Petroleum University, Chengdu 610500, China \\ ${ }^{2}$ Department of Mathematics, Zhejiang University, Hangzhou 310027, China \\ ${ }^{3}$ Department of Mathematics, College of Science, Southwest Petroleum University, Chengdu 610500, China
}

Correspondence should be addressed to Wenjun Xiong; xwenjun2@gmail.com

Received 23 October 2013; Revised 23 December 2013; Accepted 29 December 2013; Published 17 February 2014

Academic Editor: Stanislaw Migorski

Copyright (C) 2014 Ping Guo et al. This is an open access article distributed under the Creative Commons Attribution License, which permits unrestricted use, distribution, and reproduction in any medium, provided the original work is properly cited.

\begin{abstract}
The exact boundary controllability of a class of enhanced oil recovery systems is discussed in this paper. With a simple transformation, the enhanced oil recovery model is first affirmed to be neither genuinely nonlinear nor linearly degenerate. It is then shown that the enhanced oil recovery system with nonlinear boundary conditions is exactly boundary controllable by applying a constructed method. Moreover, an interval of the control time is presented to not only give the optimal control time but also show the time for avoiding the blowup of the controllable solution. Finally, an example is given to illustrate the effectiveness of the proposed criterion.
\end{abstract}

\section{Introduction}

In recent years, the economy has developed rapidly over the years requiring a lot of energy sources in China, but it is impossible to largely import oil required. Many oil fields in China are developed by water flooding, but now, the recovery efficiency is low and water cut is over $80 \%$ because of the heterogeneity of reservoirs and high viscosity of oil. It is essential to increase the oil production of oil fields. As a result, enhanced oil recovery (EOR) has been a challenging field for different scientific disciplines. A mathematical model in [1] is developed to describe surfactant-enhanced solubilization of nonaqueous-phase liquids (NAPLs) in porous media. The goal in [2] is to find an optimal viscosity profile of the intermediate layer that almost eliminates the growth of the interfacial disturbances induced by mild perturbation of the permeability field. The mechanism of enhanced oil recovery using lipophobic and hydrophilic polysilicon (LHP) nanoparticles ranging in size from 10 to $500 \mathrm{~nm}$ for changing the wettability of porous media is analyzed theoretically in [3]. It is shown in [4] that water-soluble hydrophobically associating polymers are reviewed with particular emphasis on their application in improved oil recovery (IOR). The solution properties of enhanced oil recovery are provided in $[5,6]$. In order to enhance oil recovery and stabilize oil production, the study on EOR has been carried out for more than 20 years (also see [7-11]).

One of the strategies used in EOR is to use polymer flooding. Polymer flooding involves using a polymer additive to increase water viscosity, improve the water-oil mobility ratio, and enhance the displacement efficiency. Polymer flooding has been widely applied as an effective tertiary oilrecovery method in Daqing, Shengli, and other oilfields in China.

However, Different polymer flooding units have different static conditions and development status before polymer flooding. The production performance and behavior are also different. The quantitative characterization and prediction of polymer flooding performance have important guiding significance for polymer flooding scheme programming, performance evaluation, and adjustment. Hence, it is necessary to construct some mathematical models to illustrate the properties of polymer flooding. In [12-15], a $2 \times 2$ nonlinear 
system model is presented to describe the polymer flooding of an oil recovery:

$$
\begin{gathered}
s_{t}+(s f(s, c s))_{x}=0, \\
(s c)_{t}+(s c f(s, c s))_{x}=0
\end{gathered}
$$

where $s=s(x, t)$ is the saturation of the aqueous phase (i.e., the solution of polymer and water, $0 \leq s \leq 1), c=c(x, t)$ is the concentration of polymer in the water $(0 \leq c \leq 1)$. $f=f(s, c s)[16]$ is the particle velocity of the aqueous phase. $x$ denotes the position in the reservoir and $t$ denotes the time. In the polymer flooding, water thickened with polymer is injected into the reservoir.

Let $u=(s, c s)$; system (1) can be written as

$$
u_{t}+(u f(u))_{x}=0
$$

where $u \triangleq\left(u_{1}, u_{2}\right)=(s, s c), f(u)=f(s, c s)$ is a scalar function and usually referred to be the flow function. In this paper, we consider $f$ to be rotationally invariant; namely, define that $f(u)=f(\|u\|)$ with $\|u\|=\sqrt{u_{1}^{2}+u_{2}^{2}}$. As a result, system (2) can be described as

$$
u_{t}+(u f(\|u\|))_{x}=0 .
$$

To the authors' knowledge, seldom researchers discussed the optimal control problem of system (3) (or (1)), but it is really interesting. Actually, in the last forty years, different optimal control schemes such as pinning control and impulsive control have been presented on all kinds of mathematical models of the engineering and physical application [17-19]. It is worth noting that almost all of the discussed models in [17-19] are ordinary differential but system (3) is partial differential. A problem is arisen: how to discuss the control problem of the partial differential model (3)? By the constructive method, the authors in $[20,21]$ discuss the global exact boundary controllability of a class of quasilinear hyperbolic systems of conservation laws with linearly degenerate characteristics. Inspired by [20, 21], we will discuss the exact boundary control problem of system (3) by using a constructed method. Hence, the main concern of this paper is to design an exact boundary controller for the EOR model (3).

The remainder of this paper is organized as follows. In Section 2, the exact boundary control problem and some Lemmas are presented. In Section 3, the main result is completed by a constructive method. Moreover, some important lemmas are also proposed in this section. In Section 4, an example is carried out to illustrate the effectiveness of the main result. Finally, conclusions are drawn in Section 5.

\section{Problem Description}

Let $u=R \cdot \vec{\theta}(0 \leq R \leq 1$ and $\vec{\theta}=(\cos \theta, \sin \theta)$ is a unit vector $)$; system (3) can be written as

$$
(R \vec{\theta})_{t}+(f(R) R \vec{\theta})_{x}=0
$$

one has

$$
\left(R_{t}+(f(R) R)_{x}\right) \vec{\theta}+R\left(\vec{\theta}_{t}+f(R) \vec{\theta}_{x}\right)=0 .
$$

According to (5), one has

$$
\begin{aligned}
& \left(R_{t}+(f(R) R)_{x}\right) \cos \theta-R \sin \theta\left(\theta_{t}+f(R) \theta_{x}\right)=0, \\
& \left(R_{t}+(f(R) R)_{x}\right) \sin \theta+R \cos \theta\left(\theta_{t}+f(R) \theta_{x}\right)=0 .
\end{aligned}
$$

Then, one has from (6) and (7)

$$
R_{t}+(f(R) R)_{x}=0 .
$$

Inserting (8) into (6) or (7), one gets

$$
\theta_{t}+f(R) \theta_{x}=0
$$

Hence, one has

$$
\begin{gathered}
R_{t}+A(R) R_{x}=0, \\
\theta_{t}+f(R) \theta_{x}=0,
\end{gathered}
$$

where $A(R)=f^{\prime}(R) R+f(R)$.

In the following, we will investigate the exact boundary control problem for system (10) (or system (3)). Consider system (10) posed on the domain

$$
D=\{(x, t) \mid 0 \leq x \leq 1, t \geq 0\},
$$

with the initial data

$$
R(x, 0)=R_{0}(x), \quad \theta(x, 0)=\theta_{0}(x),
$$

and the nonlinear boundary conditions

$$
\begin{aligned}
& R=g_{1}(\theta, t)+h_{1}(t), \quad \text { at } x=0, \\
& \theta=g_{2}(R, t)+h_{2}(t), \quad \text { at } x=1,
\end{aligned}
$$

where $g_{i}(R, \theta, t)(i=1,2)$ are given smooth functions. systems (10) and (13) can be viewed as boundary control systems when boundary value functions $h_{1}$ and $h_{2}$ are considered as control inputs. Hence, we only need to study the following problem.

Exact Boundary Control Problem: given $R_{0}, \theta_{0} \in C^{1}[0,1]$, and $R_{T}, \theta_{T} \in C^{1}[0,1]$, can one find a time $T>0$ and control inputs $h_{1}, h_{2} \in C^{1}[0, T]$ such that the boundary control systems (10) and (13) have a $C^{1}$ solution $(R, \theta)$ satisfying the initial conditions (12) and the terminal conditions

$$
R(x, T)=R_{T}(x), \quad \theta(x, T)=\theta_{T}(x) ?
$$

In order to solve the above boundary control problem, we need the following assumptions and Lemmas.

Assumption 1. For any $R \in[0,1]$, there exists

$$
f(R)<0<A(R) .
$$


Assumption 2. For simplification, we assume that

$$
\frac{\partial A(R)}{\partial R}>0, \quad \forall R \in[0,1] .
$$

As a result, when $R_{0}^{\prime}(x) \geq 0$, the Cauchy problem (10) and (12) has a $C^{1}$ global solution. Moreover, $R_{x} \geq 0$.

Remark 3. Assumption 1 denotes that system (10) is strongly strictly hyperbolic.

Remark 4. The discussed models in $[20,21]$ are both linearly degenerate. However, model (10) in this paper is neither genuinely nonlinear nor linearly degenerate, which is more difficult and complicated to be discussed.

Lemma 5 (see [22]). Consider the Cauchy problem (10) and (12). Suppose that $f(R), A(R), R_{0}, \theta_{0}$ are all $C^{1}$ functions and the $C^{1}$ norm of $R_{0}(x)$ and $\theta_{0}(x)$ are bounded. Under Assumption 1, if

$$
\frac{\partial A\left(R_{0}(\alpha)\right)}{\partial \alpha} \geq 0, \quad \forall \alpha \in[0,1],
$$

the Cauchy problem (10) and (12) has a unique global $C^{1}$ solution $(R, \theta)=(R(t, x), \theta(t, x))$ on the domain $\Gamma=\{(t, x) \mid$ $x \in \mathbb{R}, t \geq 0\}$.

Remark 6. For the Cauchy problem (10) and (14), we need to modify (17) to be

$$
\frac{\partial A\left(R_{T}(\beta)\right)}{\partial \beta} \leq 0, \quad \forall \beta \in[0,1] .
$$

When $R_{T}^{\prime}(x) \leq 0$, the Cauchy problem (10) and (14) has a unique global $C^{1}$ solution $(R, \theta)=(R(t, x), \theta(t, x))$ on the domain $\Gamma_{1}=\{(t, x) \mid x \in[0,1], t \in(-\infty, T]\}$. Moreover, $R_{x} \leq 0$.

In the following, we consider the Goursat problem of system (10) on the angular domain

$$
D_{1}=\left\{(x, t) \mid x_{1}(t) \leq x \leq x_{2}(t), t \geq 0\right\} .
$$

We prescribe boundary conditions

$$
\begin{aligned}
& R\left(t, x_{1}(t)\right)=\widetilde{R}(t), \quad \text { on } x=x_{1}(t), \\
& \theta\left(t, x_{2}(t)\right)=\widetilde{\theta}(t), \quad \text { on } x=x_{2}(t),
\end{aligned}
$$

where $x_{1}(t)$ and $x_{2}(t)$ are the characteristics passing through the origin point $O=(0,0)$, on which it holds

$$
\begin{array}{ll}
\frac{d x_{1}(t)}{d t}=f(\widetilde{R}(t)), & x_{1}(0)=0, \\
\frac{d x_{2}(t)}{d t}=A\left(R_{0}(0)\right), & x_{2}(0)=0 .
\end{array}
$$

Lemma 7 (see [22]). Suppose that $f(R), A(R), \widetilde{R}, \widetilde{\theta}$ are all $C^{1}$ functions. Under Assumptions 1 and 2, if

$$
\widetilde{R}^{\prime}(t) \leq 0 \text {, }
$$

the Goursat problem (10) and (20) has a unique global $C^{1}$ solution $(\widetilde{R}, \widetilde{\theta})$ on the domain $D_{1}$.

First, we need to discuss the lifespan of the Cauchy problem and Goursat problem. From the Cauchy problem (10) and (12), we have the following lemma.

Lemma 8. If there exists $\alpha_{0} \in \mathbb{R}$ such that $d R_{0}(\alpha) /\left.d \alpha\right|_{\alpha=\alpha_{0}}<$ 0 , the Cauchy problem (10) and (12) must blow up in a finite time and the lifespan is dependent on the initial data.

Proof. For the first equation of system (10), the characteristic $x_{2}(t)$ can be defined by

$$
\frac{d x_{2}(t)}{d t}=A\left(R\left(x_{2}(t), t\right)\right), \quad x_{2}(0)=\alpha
$$

One has $d R / d t=0$; that is, $R\left(x_{2}(t), t\right)=R_{0}(\alpha)$. That is, the $C^{0}$ norm of $R$ is finite. Hence, we need to show that the first derivative of $R$ must blow up in a finite time.

Clearly, $R_{x}=\left(d R_{0}(\alpha) / d \alpha\right) /(d x / d \alpha)$. From (23), one has $x_{2}=\alpha+A\left(R_{0}(\alpha)\right) t$. Then,

$$
\frac{d x_{2}}{d \alpha}=1+\frac{\partial A(R)}{\partial R} \frac{d R_{0}(\alpha)}{d \alpha} t
$$

According to (16), if there exists $\alpha_{0} \in \mathbb{R}$ such that $d R_{0}(\alpha) /\left.d \alpha\right|_{\alpha=\alpha_{0}}<0$, one has

$$
R_{x} \longrightarrow \infty, \quad \text { at } t=\frac{-1}{\left.(\partial A(R) / \partial R)\left(d R_{0}(\alpha) / d \alpha\right)\right|_{\alpha=\alpha_{0}}}
$$

That is, the Cauchy problem (10) and (12) must blow up in a finite time. Moreover, from (24), one has $t=1 / O(\varepsilon)$ when $d R_{0}(\alpha) / d \alpha=O(\varepsilon)$, which means that the lifespan $t$ is dependent on the initial data. The proof is completed.

Remark 9. Note that, in the second equation of system (10), according to [22], $\theta_{x}$ will always be bounded. Moreover, $\theta_{x}$ will blow up if the following holds:

$$
\frac{\partial f\left(R_{0}(\alpha), \theta_{0}(\beta)\right)}{\partial \beta}=\frac{\partial f}{\partial \theta} \cdot \frac{\partial \theta_{0}(\beta)}{\partial \beta}<0 .
$$

Obviously, this does not hold since $f(\cdot)$ is independent of the function $\theta$. As a result, $\theta_{x}$ will never blow up in a finite time.

For the Goursat problem (10) and (20), we have the following result.

Lemma 10. If there exists $\alpha_{0} \in \mathbb{R}$ such that $\widetilde{R}^{\prime}\left(\alpha_{0}\right)>0$, the solution of the Goursat problem (10) and (20) must blow up in a finite time and the lifespan depends on the initial data.

Proof. For $\forall(x, t) \in D_{1}$, its two characteristics have two intersect points with the curves $x_{1}(t)$ and $x_{2}(t)$, which are, respectively, defined as $\left(x_{1}(\alpha), \alpha\right)$ and $\left(x_{2}(\beta), \beta\right)$. Along the two characteristics, one has $R(x, t)=\widetilde{R}(\alpha)$ and $\theta(x, t)=\widetilde{\theta}(\beta)$, respectively. As a result, $R(x, t), \theta(x, t)$ are bounded.

In the following, we will calculate $R_{x}$ and $\theta_{x}$. Clearly, one has $R_{x}=(d \widetilde{R}(\alpha) / d \alpha) /(d x / d \alpha)$, and $x(t)=$ 
$x_{1}(\alpha)+A(\widetilde{R}(\alpha))(t-\alpha)$. Hence, $x_{\alpha}=f(\widetilde{R}(\alpha))+$ $(\partial A(\widetilde{R}(\alpha)) / \partial R)(d \widetilde{R}(\alpha) / d \alpha)(t-\alpha)-A(\widetilde{R}(\alpha))$. According to (15) and (16), one has the fact that $f(\widetilde{R}(\alpha))-A(\widetilde{R}(\alpha))<0$ and $\partial A(\widetilde{R}(\alpha)) / \partial R>0$. As a result, if there exists $\alpha_{0} \in \mathbb{R}$ such that $\widetilde{R}^{\prime}\left(\alpha_{0}\right)>0, R_{x} \rightarrow \infty$ in a finite time $t$. Moreover, similar to Lemma 8, one knows that the lifespan $t$ depends on the initial data.

Similarly, one has $\theta(x, t)=\tilde{\theta}(\beta)$ with $x=x_{2}(\beta)+$ $f(\widetilde{R}(\alpha))(t-\beta)$. As a result, $d x / d \beta=A(\widetilde{R}(\alpha))-f(\widetilde{R}(\alpha))>0$, which means that $\theta_{x}$ will never blow up in a finite time.

Remark 11. According to Lemmas 8 and 10 and Remark 9, one knows that the blowup of the Goursat problem and the Cauchy problem only occurs in the solution $R$.

\section{Main Results}

In this section, the boundary controllers will be designed.

Theorem 12. With Assumptions 1 and 2, and conditions (17)(18), for given $R_{0}, \theta_{0}, R_{T}, \theta_{T}$ in the space $C^{1}[0,1]$ with their $C^{0}$ norms bounded by $\Xi>0$ and the small norms of their first derivatives, and for any $T$ satisfying $T_{0}<T<T_{1}<+\infty$, there exist $h_{1}(t), h_{2}(t) \in C^{1}[0, T]$ such that systems (10) and (12) have a $C^{1}$ solution $(R(x, t), \theta(x, t))$ on the domain

$$
D_{T}=\{(x, t) \mid 0 \leq x \leq 1,0 \leq t \leq T\},
$$

satisfying $R(x, 0)=R_{0}(x), \theta(x, 0)=\theta_{0}(x), R(x, T)=R_{T}(x)$, $\theta(x, T)=\theta_{T}(x)$, for $0 \leq x \leq 1$, where

$$
T_{0}=\max \left\{-\frac{1}{f_{\max }}, \frac{1}{A_{\min }}\right\},
$$

and $f_{\max }=\max _{|R| \leq \Xi} f(R), A_{\min }=\min _{|R| \leq \Xi} A(R)$. Moreover, $T_{1}$ is the lifespan of the Cauchy problem (10) with the initial data on $t=T$.

Proof. One has the following.

Step 1. Discuss $T_{0}$. Let $O=(0,0), A(1,0), B=(1, T), C=$ $(0, T)$ (see Figure 1). Let $J=\left(x_{j}, t_{j}\right)$ be the intersection point of the lines

$$
l_{1}: x(t)=A_{\min } t, \quad l_{2}: x(t)=1+f_{\max } t .
$$

Let $G=\left(x_{g}, t_{g}\right)$ be the intersection point of the lines

$$
\bar{l}_{1}: x(t)=1+A_{\min }(t-T), \quad \bar{l}_{2}: x(t)=f_{\max }(t-T) .
$$

Let the curves $O P M$ and $A P F$ be, respectively, described by the characteristics $x_{1}(t)$ and $x_{2}(t)$ (see Figure 1), which satisfy

$$
\begin{gathered}
\frac{d x_{1}(t)}{d t}=f\left(R\left(x_{1}(t)\right), t\right), \quad t=0: x_{1}(0)=1, \\
\frac{d x_{2}(t)}{d t}=A\left(R_{0}(0)\right), \quad t=0: x_{2}(0)=0 .
\end{gathered}
$$

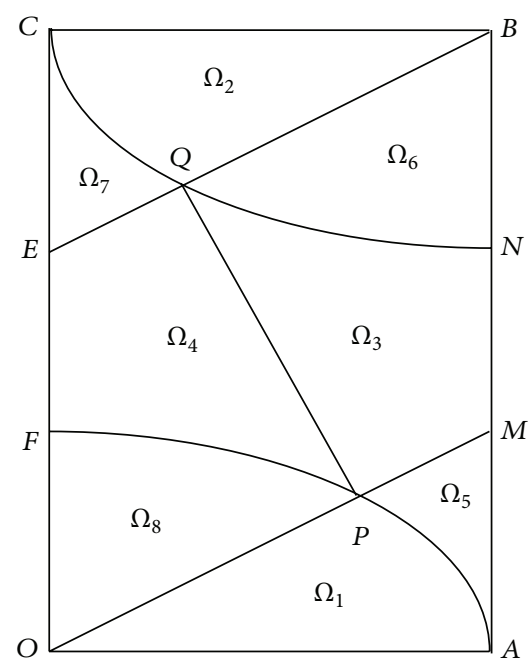

Figure 1: Domains $\Omega_{i}(i=1,2, \ldots, 8)$, characteristics: OPM: $x=$ $x_{2}(t), A P F: x=x_{1}(t)$, CQN: $x=\bar{x}_{1}(t), B Q E: x=$ $\bar{x}_{2}(t)$, straight line: $P Q$.

Define

$$
\widetilde{R}(t) \equiv R\left(x_{2}(t), t\right), \quad \widetilde{\theta}(t) \equiv \theta\left(x_{1}(t), t\right) .
$$

Let $P=\left(x_{p}, t_{p}\right)$ be the intersection point of the curves OPM and $A P F$. Similarly, let $Q=\left(x_{q}, t_{q}\right)$ be the intersection point of the curves $C Q N$ and $B Q E$. Here, curves $C Q N$ and $B Q E$ are, respectively, described by the characteristics $\bar{x}_{1}(t)$ and $\bar{x}_{2}(t)$ (see Figure 1), which satisfy

$$
\begin{gathered}
\frac{d \bar{x}_{1}(t)}{d t}=f\left(R\left(\bar{x}_{1}(t), t\right)\right), \quad t=T: \bar{x}_{1}(T)=0, \\
\frac{d \bar{x}_{2}(t)}{d t}=A\left(R_{T}(1)\right), \quad t=T: \bar{x}_{2}(T)=1 .
\end{gathered}
$$

Define

$$
\bar{R}(t) \equiv R\left(\bar{x}_{2}(t), t\right), \quad \bar{\theta}(t) \equiv \theta\left(\bar{x}_{1}(t), t\right) .
$$

Here, we have to satisfy two conditions.

(1) The lines $l_{1}$ and $B C$ have no intersection point in $x \in$ $[0,1]$.

(2) The lines $l_{2}$ and $B C$ have no intersection point in $x \in$ $[0,1]$.

Otherwise, if condition (1) is not satisfied, there exists a characteristic $x=\widehat{x}_{2}(t)$ which passes through two points $O=(0,0)$ and $C_{1}=\left(\alpha_{1}, T\right), 0 \leq \alpha_{1} \leq 1$. According to the characteristic property, one has $R(x, t)=R_{0}(0)=R_{T}\left(\alpha_{1}\right)$, for $\forall(t, x) \in \widehat{\Omega}$ and $\widehat{\Omega}$ is enclosed by the characteristics $x=x_{1}(t)$, $x=\widehat{x}_{2}(t)$, and the $x$ axis.

Note that the initial and terminal conditions are usually to be arbitrarily chosen. If one choose that $R_{0}(0) \neq R_{T}\left(\alpha_{1}\right)$, the system will not go from the given initial state to the desired terminal state no matter what control inputs are given. As a result, condition (1) should be satisfied. Also, with the similar analysis, condition (2) should be satisfied. 
Let $M_{1}$ be the intersection point of the lines $l_{1}$ and $A B$; one has $M_{1}=\left(1,1 / A_{\text {min }}\right)$. From condition (1), one has the fact that $1 / A_{\min }<T$. Let $N_{1}$ be the intersection point of the lines $l_{2}$ and $O C$; one has $N_{1}=\left(0,-1 / f_{\max }\right)$. From condition (2), one has that $-1 / f_{\max }<T$. As a result, $T>\max \left\{1 / A_{\min },-1 / f_{\max }\right\}$. Hence, $T>T_{0}$ and $T_{0}=$ $\max \left\{1 / A_{\text {min }},-1 / f_{\text {max }}\right\}$.

Remark 13. (i) In $[20,21]$, the authors require that $T_{0}=$ $\max \left\{1 / A_{\min },-1 / f_{\max }, 2 /\left(A_{\min }-f_{\max }\right)\right\}$. Actually, we only need that $T_{0}=\max \left\{1 / A_{\min },-1 / f_{\max }\right\}$. Here, $J=\left(x_{j}, t_{j}\right)=$ $\left(A_{\min } /\left(A_{\min }-f_{\max }\right), 1 /\left(A_{\min }-f_{\max }\right)\right)$, and $G=\left(x_{g}, t_{g}\right)=$ $\left(-f_{\max } /\left(A_{\min }-f_{\max }\right), T-\left(1 /\left(A_{\min }-f_{\max }\right)\right)\right)$.

If $1 / A_{\min }<1 /-f_{\max }$, then $A_{\min }>-f_{\max }, T_{0}=1 /-f_{\max }$ and $T>T_{0}$. One has the fact that

$$
\begin{aligned}
t_{g}-t_{j} & =T-\frac{2}{A_{\min }-f_{\max }}>\frac{1}{-f_{\max }}-\frac{2}{A_{\min }-f_{\max }} \\
& =-\frac{A_{\min }+f_{\max }}{f_{\max }\left(A_{\min }-f_{\max }\right)}>0 .
\end{aligned}
$$

From the above inequality, one has $1 /-f_{\max }>2 /\left(A_{\min }-\right.$ $\left.f_{\max }\right)$. Similarly, if $1 / A_{\min }>1 /-f_{\max }$, one can obtain that $1 / A_{\min }>2 /\left(A_{\min }-f_{\max }\right)$.

Hence, $T_{0}=\max \left\{1 / A_{\min },-1 / f_{\max }, 2 /\left(A_{\min }-f_{\max }\right)\right\}$ can be written as $T_{0}=\max \left\{1 / A_{\min },-1 / f_{\max }\right\}$. (ii) $T<T_{1}$ denotes that the solution of the Cauchy problem does not blow up in the domain $\Omega_{2}$.

Step 2. Discuss the Cauchy problem in the domains $\Omega_{1}$ and $\Omega_{2}$. Let the domain $\Omega_{1}$ be enclosed by the characteristics $x_{1}(t)$ and $x_{2}(t)$ and the $x$ axis (see Figure 1). Let the domain $\Omega_{2}$ be enclosed by the characteristics $\bar{x}_{1}(t)$ and $\bar{x}_{2}(t)$ and the horizontal line $t=T$ (see Figure 1). From Lemma 5 and Remark 6, the Cauchy problem (10) and (12) (or (10) and (14)) has a unique global $C^{1}$ solution $(R, \theta)=(R(t, x), \theta(t, x))$ on the domain $\Omega_{1}$ (or $\left.\Omega_{2}\right)$. Moreover, one has

(1) on the straight line $O P M, R=R_{0}(0)$, and on the curve $A P F, \theta=\theta_{0}(1)$;

(2) on the straight line $B Q E, R=R_{T}(1)$, and on the curve CQN, $\theta=\theta_{T}(0)$.

Step 3. Let $\Omega_{3}$ be the domain enclosed by the characteristic $P M$, the characteristic $Q N$, the straight line $M N$, and the straight line $P Q$, where $P Q$ is denoted by

$$
x(t)=x_{3}(t)=x_{p}+k\left(t-t_{p}\right), \quad t_{p} \leq t \leq t_{q},
$$

where $k=\left(x_{q}-x_{p}\right) /\left(t_{q}-t_{p}\right)$ is the slope $d x / d t$ of the straight line $P Q$. Consider the following system on the domain $\Omega_{3}$ :

$$
\begin{aligned}
& R_{x}+\frac{1}{A(R)} R_{t}=0, \\
& \theta_{x}+\frac{1}{f(R)} \theta_{t}=0,
\end{aligned}
$$

with initial conditions

$$
\begin{gathered}
\widetilde{R}_{0}(t)=R\left(x_{3}(t), t\right), \\
\widetilde{\theta}_{0}(t)=\theta\left(x_{3}(t), t\right) .
\end{gathered}
$$

For points $P$ and $Q$, it is required that

$$
\begin{aligned}
& \widetilde{R}_{0}\left(t_{q}\right)=R\left(\bar{x}_{2}\left(t_{q}\right), t_{q}\right)=R_{T}(1), \\
& \widetilde{\theta}_{0}\left(t_{q}\right)=\theta\left(\bar{x}_{1}\left(t_{q}\right), t_{q}\right)=\theta_{T}(0), \\
& \widetilde{R}_{0}\left(t_{p}\right)=R\left(x_{2}\left(t_{p}\right), t_{p}\right)=R_{0}(0), \\
& \widetilde{\theta}_{0}\left(t_{p}\right)=\theta\left(x_{1}\left(t_{p}\right), t_{p}\right)=\theta_{0}(1) .
\end{aligned}
$$

Along the characteristic $Q N: x=\bar{x}_{1}(t)$, one has

$$
\begin{aligned}
\bar{R}^{\prime}\left(t_{q}\right) & =\left.\frac{d R\left(\bar{x}_{1}(t), t\right)}{d t}\right|_{t=t_{q}}=\left.\left(\frac{\partial R}{\partial t}+\frac{\partial R}{\partial x} f(R)\right)\right|_{t=t_{q}} \\
& =\left.(f(R)-A(R)) \frac{\partial R}{\partial x}\right|_{t=t_{q}} \\
& =\left(f\left(R_{T}(1)\right)-A\left(R_{T}(1)\right)\right) \frac{\partial R}{\partial x} .
\end{aligned}
$$

Along the straight line $P Q$, one has

$$
\begin{aligned}
\widetilde{R}_{0}^{\prime}\left(t_{q}\right) & =\left.\frac{d R\left(x_{3}(t), t\right)}{d t}\right|_{t=t_{q}}=\left.\left(\frac{\partial R}{\partial t}+\frac{\partial R}{\partial x} k\right)\right|_{t=t_{q}} \\
& =\left.(k-A(R)) \frac{\partial R}{\partial x}\right|_{t=t_{q}}=\left(k-A\left(R_{T}(1)\right)\right) \frac{\partial R}{\partial x} .
\end{aligned}
$$

So, it is required that

$$
\widetilde{R}_{0}^{\prime}\left(t_{q}\right)=\bar{R}^{\prime}\left(t_{q}\right) \frac{k-A\left(R_{T}(1)\right)}{f\left(R_{T}(1)\right)-A\left(R_{T}(1)\right)} .
$$

Similarly, along the characteristic $Q E: x=\bar{x}_{2}(t)$, one has

$$
\begin{aligned}
\bar{\theta}^{\prime}\left(t_{q}\right) & =\left.\frac{d \theta\left(\bar{x}_{2}(t), t\right)}{d t}\right|_{t=t_{q}}=\left.\left(\frac{\partial \theta}{\partial t}+\frac{\partial \theta}{\partial x} A(R)\right)\right|_{t=t_{q}} \\
& =\left.(A(R)-f(R)) \frac{\partial \theta}{\partial x}\right|_{t=t_{q}} \\
& =\left(A\left(R_{T}(1)\right)-f\left(R_{T}(1)\right)\right) \frac{\partial \theta}{\partial x} .
\end{aligned}
$$

Along the straight line $P Q$, one has

$$
\begin{aligned}
\tilde{\theta}_{0}^{\prime}\left(t_{q}\right) & =\left.\frac{d \theta\left(x_{3}(t), t\right)}{d t}\right|_{t=t_{q}}=\left.\left(\frac{\partial \theta}{\partial t}+\frac{\partial \theta}{\partial x} k\right)\right|_{t=t_{q}} \\
& =\left.(k-f(R)) \frac{\partial \theta}{\partial x}\right|_{t=t_{q}}=\left(k-f\left(R_{T}(1)\right)\right) \frac{\partial \theta}{\partial x} .
\end{aligned}
$$


It is therefore required that

$$
\widetilde{\theta}_{0}^{\prime}\left(t_{q}\right)=\bar{\theta}^{\prime}\left(t_{q}\right) \frac{k-f\left(R_{T}(1)\right)}{A\left(R_{T}(1)\right)-f\left(R_{T}(1)\right)}
$$

In addition, along the characteristic $P F: x=x_{1}(t)$, one has

$$
\begin{aligned}
\widetilde{R}^{\prime}\left(t_{p}\right) & =\left.\frac{d R\left(x_{1}(t), t\right)}{d t}\right|_{t=t_{p}}=\left.\left(\frac{\partial R}{\partial t}+\frac{\partial R}{\partial x} f(R)\right)\right|_{t=t_{p}} \\
& =\left.(f(R)-A(R)) \frac{\partial R}{\partial x}\right|_{t=t_{p}} \\
& =\left(f\left(R_{0}(0)\right)-A\left(R_{0}(0)\right)\right) \frac{\partial R}{\partial x}
\end{aligned}
$$

Along the straight line $P Q$, one has

$$
\begin{aligned}
\widetilde{R}_{0}^{\prime}\left(t_{p}\right) & =\left.\frac{d R\left(x_{3}(t), t\right)}{d t}\right|_{t=t_{p}}=\left.\left(\frac{\partial R}{\partial t}+\frac{\partial R}{\partial x} k\right)\right|_{t=t_{p}} \\
& =\left.(k-A(R)) \frac{\partial R}{\partial x}\right|_{t=t_{p}}=\left(k-A\left(R_{0}(0)\right)\right) \frac{\partial R}{\partial x}
\end{aligned}
$$

So, it is required that

$$
\widetilde{R}_{0}^{\prime}\left(t_{p}\right)=\widetilde{R}^{\prime}\left(t_{p}\right) \frac{k-A\left(R_{0}(0)\right)}{f\left(R_{0}(0)\right)-A\left(R_{0}(0)\right)}
$$

Similarly, along the characteristic $P M: x=x_{2}(t)$, one has

$$
\begin{aligned}
\tilde{\theta}^{\prime}\left(t_{p}\right) & =\left.\frac{d \theta\left(x_{2}(t), t\right)}{d t}\right|_{t=t_{p}}=\left.\left(\frac{\partial \theta}{\partial t}+\frac{\partial \theta}{\partial x} A(R)\right)\right|_{t=t_{p}} \\
& =\left.(A(R)-f(R)) \frac{\partial \theta}{\partial x}\right|_{t=t_{p}} \\
& =\left(A\left(R_{0}(0)\right)-f\left(R_{0}(0)\right)\right) \frac{\partial \theta}{\partial x}
\end{aligned}
$$

Along the straight line $P Q$, one has

$$
\begin{aligned}
\widetilde{\theta}_{0}^{\prime}\left(t_{p}\right) & =\left.\frac{d \theta\left(x_{3}(t), t\right)}{d \mathrm{t}}\right|_{t=t_{p}}=\left.\left(\frac{\partial \theta}{\partial t}+\frac{\partial \theta}{\partial x} k\right)\right|_{t=t_{p}} \\
& =\left.(k-f(R)) \frac{\partial \theta}{\partial x}\right|_{t=t_{p}}=\left(k-f\left(R_{0}(0)\right)\right) \frac{\partial \theta}{\partial x}
\end{aligned}
$$

It is therefore required that

$$
\widetilde{\theta}_{0}^{\prime}\left(t_{p}\right)=\widetilde{\theta}^{\prime}\left(t_{p}\right) \frac{k-f\left(R_{0}(0)\right)}{A\left(R_{0}(0)\right)-f\left(R_{0}(0)\right)}
$$

Moreover, the following compatibility conditions of points $O$, $A, B, C$ are also required:

$$
\begin{aligned}
& R_{0}(0)=g_{1}\left(\theta_{0}(0), 0\right)+h_{1}(0), \\
& \theta_{0}(1)=g_{2}\left(R_{0}(1), 0\right)+h_{2}(0), \\
& R_{T}(0)=g_{1}\left(\theta_{T}(0), T\right)+h_{1}(T), \\
& \theta_{T}(1)=g_{2}\left(R_{T}(1), T\right)+h_{2}(T), \\
& -A\left(R_{0}(0)\right) R_{0}^{\prime}(0)=\frac{\partial g_{1}\left(\theta_{0}(0), 0\right)}{\partial t} \\
& -f\left(R_{0}(0)\right) \frac{\partial g_{1}\left(\theta_{0}(0), 0\right)}{\partial \theta} \theta_{0}^{\prime}(0) \\
& +h_{1}^{\prime}(0) \text {, } \\
& -A\left(R_{T}(0)\right) R_{T}^{\prime}(0)=\frac{\partial g_{1}\left(\theta_{T}(0), T\right)}{\partial t} \\
& -f\left(R_{T}(0)\right) \frac{\partial g_{1}\left(\theta_{T}(0), T\right)}{\partial \theta} \theta_{T}^{\prime}(0) \\
& +h_{1}^{\prime}(T) \text {, } \\
& -f\left(R_{0}(1)\right) \theta_{0}^{\prime}(1)=\frac{\partial g_{2}\left(R_{0}(1), 0\right)}{\partial t} \\
& -A\left(R_{0}(1)\right) \frac{\partial g_{2}\left(R_{0}(1), 0\right)}{\partial R} R_{0}^{\prime}(1) \\
& +h_{2}^{\prime}(0) \text {, } \\
& -f\left(R_{T}(1)\right) \theta_{T}^{\prime}(1)=\frac{\partial g_{2}\left(R_{T}(1), T\right)}{\partial t} \\
& -A\left(R_{T}(1)\right) \frac{\partial g_{2}\left(R_{T}(1), T\right)}{\partial R} R_{T}^{\prime}(1) \\
& +h_{2}^{\prime}(T)
\end{aligned}
$$

According to Proposition 2.1 of [21], one has the fact that

$$
f(R)<k<A(R), \quad t_{p} \leq t \leq t_{q},
$$

when $T>T_{0}=\max \left\{-1 / f_{\max }, 1 / A_{\min }\right\}$. Then, from Assumption 2 and Remark 6, one has $\widetilde{R}_{0}^{\prime}\left(t_{q}\right)>0, \widetilde{R}_{0}^{\prime}\left(t_{p}\right)<$ 0 . With Lemma 8 , the Cauchy problem (37) and (38) with prescribed data on $x_{3}(t)$ must blow up in a finite time. Here, we have interchanged the role of $x$ and $t$ variables. Hence, the time means the $x$-axis.

In what follows we will prove that we can choose an appropriate vector $\left(\widetilde{R}_{0}(t), \widetilde{\theta}_{0}(t)\right)$ to satisfy that the first blowup point is out of $(0,1)$.

Lemma 14. One can choose $\left(\widetilde{R}_{0}(t), \widetilde{\theta}_{0}(t)\right)$ such that the first blowup point $(x, t)$ satisfying $x>1$; that is, the Cauchy problem (37) and (38) has $C^{1}$ solution on the domain $\Omega_{3}$. 
Proof. $\forall(x, t) \in \Omega_{3}$, two characteristics passing by $(x, t)$ can be defined by

$$
\begin{array}{ll}
\frac{d t_{1}(x)}{d x}=\frac{1}{A\left(R\left(x, t_{1}(x)\right)\right)}, & t_{1}\left(x_{3}(\widetilde{\beta})\right)=\tilde{\beta}, \\
\frac{d t_{2}(x)}{d x}=\frac{1}{f\left(R\left(x, t_{2}(x)\right)\right)}, & t_{2}\left(x_{3}(\widetilde{\alpha})\right)=\tilde{\alpha},
\end{array}
$$

where $\left(x_{3}(\widetilde{\alpha}), \widetilde{\alpha}\right)$ and $\left(x_{3}(\widetilde{\beta}), \widetilde{\beta}\right)$ are the intersection points of the two characteristics and the straight line $P Q$. Along the characteristic $t_{1}(x)$, one has the fact that $d R / d x=0$. As a result,

$$
R(x, t)=\widetilde{R}_{0}(\widetilde{\beta}) .
$$

From (54), one has $t_{1}=\widetilde{\beta}+\left(1 / A\left(\widetilde{R}_{0}(\widetilde{\beta})\right)\right)\left(x-x_{3}(\widetilde{\beta})\right)$. According to Lemma 10 and Remark 9, one knows that $\theta(x, t)$ never blow up. Hence, we only need to estimate $R_{t}$. From (56), one has $R_{t}=\widetilde{R}_{0}^{\prime}(\widetilde{\beta}) /\left(d t_{1} / d \widetilde{\beta}\right)$ and $d t_{1} / d \widetilde{\beta}=1-$ $\left(x-x_{3}(\widetilde{\beta})\right)\left(\partial A\left(\widetilde{R}_{0}(\widetilde{\beta})\right) / \partial \widetilde{\beta}\right) / A^{2}\left(\widetilde{R}_{0}(\widetilde{\beta})\right)-k / A\left(\widetilde{R}_{0}(\widetilde{\beta})\right)=1-$ $k / A\left(\widetilde{R}_{0}(\widetilde{\beta})\right)-\left(x-x_{3}(\widetilde{\beta})\right)\left(\partial A\left(\widetilde{R}_{0}(\widetilde{\beta})\right) / \partial \widetilde{R}\right) \widetilde{R}_{0}^{\prime}(\widetilde{\beta}) / A^{2}\left(\widetilde{R}_{0}(\widetilde{\beta})\right)$. From (53), one can find $\delta>0$ such that $1-k / A\left(\widetilde{R}_{0}(\widetilde{\beta})\right) \geq$ $\delta>0$. Note that $x>x_{3}(\widetilde{\beta})$ in $\Omega_{3}, R_{t} \nrightarrow \infty$ if $\widetilde{R}_{0}^{\prime}(\widetilde{\beta})<0$. So, we only need to discuss $\widetilde{R}_{0}^{\prime}(\widetilde{\beta})>0$.

When $\widetilde{R}_{0}^{\prime}(\widetilde{\beta})>0$, there exists $x$ such that $R_{t} \rightarrow \infty$. As a result, if we choose $\widetilde{R}_{0}^{\prime}(\widetilde{\beta})$ satisfy that

$$
0<\widetilde{R}_{0}^{\prime}(\widetilde{\beta})<\min _{t_{p} \leq \widetilde{\beta} \leq t_{q}}\left\{\frac{A^{2}\left(\widetilde{R}_{0}(\widetilde{\beta})\right)-k A\left(\widetilde{R}_{0}(\widetilde{\beta})\right)}{\partial A\left(\widetilde{R}_{0}(\widetilde{\beta})\right) / \partial \widetilde{R}}\right\},
$$

then, the blowup point satisfies that $x>x_{3}(\widetilde{\beta})$ and $x>1$.

Remark 15. For convenience, conditions (17) and (18) are chosen to guarantee that there have been no blowup points in $\Omega_{1}$ and $\Omega_{2}$. Actually, without conditions (17) and (18), we can also get the $C^{1}$ solution in the domains $\Omega_{1}$ and $\Omega_{2}$ if we use the above method to obtain a similar condition of (57). $\Omega_{4}$.

Similarly, we have the following lemma for the domain

Lemma 16. One can choose $\left(\widetilde{R}_{0}(t), \widetilde{\theta}_{0}(t)\right)$ such that the first blowup point $(x, t)$ satisfying $x<0$, that is, the Cauchy problem (37) and (38), has $C^{1}$ solution on the domain $\Omega_{4}$.

Proof. Using the similar proof with Lemma 14, if we choose $\widetilde{R}_{0}^{\prime}\left(\widetilde{\beta_{1}}\right)$ satisfy that

$$
-\max _{t_{p} \leq \widetilde{\beta_{1}} \leq t_{q}}\left\{\frac{A^{2}\left(\widetilde{R}_{0}\left(\widetilde{\beta_{1}}\right)\right)-k A\left(\widetilde{R}_{0}\left(\widetilde{\beta_{1}}\right)\right)}{\partial A\left(\widetilde{R}_{0}\left(\widetilde{\beta_{1}}\right)\right) / \partial \widetilde{R}}\right\}<\widetilde{R}_{0}^{\prime}\left(\widetilde{\beta_{1}}\right),
$$

where $\left(x_{3}\left(\widetilde{\beta_{1}}\right), \widetilde{\beta_{1}}\right)$ is the intersection point of the straight line $P Q$ and the following characteristic

$$
\frac{d t_{1}^{1}(x)}{d x}=\frac{1}{A\left(R\left(x, t_{1}^{1}(x)\right)\right)}, \quad t_{1}^{1}\left(x_{3}\left(\widetilde{\beta_{1}}\right)\right)=\widetilde{\beta_{1}},
$$

then, the blowup point satisfies that $x<x_{3}\left(\widetilde{\beta_{1}}\right)$ and $x<0$.
Until now, we have got $C^{1}$ solutions in the domains $\Omega_{1}$, $\Omega_{2}, \Omega_{3}$, and $\Omega_{4}$ and the solutions can be defined

$$
(R(x, t), \theta(x, t))= \begin{cases}\left(R_{1}(x, t), \theta_{1}(x, t)\right), & \text { for }(x, t) \in \Omega_{1}, \\ \left(R_{2}(x, t), \theta_{2}(x, t)\right), & \text { for }(x, t) \in \Omega_{2}, \\ \left(R_{3}(x, t), \theta_{3}(x, t)\right), & \text { for }(x, t) \in \Omega_{3}, \\ \left(R_{4}(x, t), \theta_{4}(x, t)\right), & \text { for }(x, t) \in \Omega_{4} .\end{cases}
$$

Step 4. Consider the Goursat problem in $\Omega_{5}, \Omega_{6}, \Omega_{7}$, and $\Omega_{8}$. In $\Omega_{8}$, we prescribe data as follows:

$$
\begin{aligned}
& \widetilde{R}(t)=R_{4}\left(x_{1}(t), t\right), \quad \text { on } x_{1}(t), \\
& \tilde{\theta}(t)=\theta_{1}\left(x_{2}(t), t\right), \quad \text { on } x_{2}(t) .
\end{aligned}
$$

For $\forall(x, t) \in \Omega_{8}$, a characteristic passing by $(x, t)$ can be defined by

$$
\frac{d \widetilde{t}_{1}(x)}{d x}=\frac{1}{A\left(R\left(x, \widetilde{t}_{1}(x)\right)\right)}, \quad \tilde{t}_{1}\left(x_{1}(\zeta)\right)=\zeta,
$$

where $\left(x_{1}(\zeta), \zeta\right)$ is the intersection point of this characteristic and the characteristic $x_{1}(t)$. Along the characteristic $\tilde{t}_{1}(x)$, one has $d R / d x=0$; that is, $R=\widetilde{R}(\zeta)$. Clearly, the $C^{0}$ norm of $R$ is bounded. In order to estimate $R_{t}$, we need to discuss $d \tilde{t}_{1}(x) / d \zeta$. From $(62)$, one has $\tilde{t}_{1}(x)=\zeta+$ $(1 / A(R))\left(x-x_{1}(\zeta)\right)$. Then, $d \tilde{t}_{1}(x) / d \zeta=1-(f(R) / A(R))-(x-$ $\left.x_{1}(\zeta)\right)(\partial A / \partial R)(d R / d \zeta) / A^{2}(R)$. According to Assumption 2, there at least exists a point $\left(x\left(\zeta_{1}\right), \zeta_{1}\right)$ such that $R_{x}\left(\zeta_{1}\right)>0$. Then, $d R /\left.d \zeta\right|_{\zeta=\zeta_{1}}=R_{t}+\left.R_{x} f(R)\right|_{\zeta=\zeta_{1}}=(f(R)-A(R)) R_{x}\left(\zeta_{1}\right)<$ 0 . Note that $x-x_{1}(\zeta)<0$, there exists $x$ such that $R_{t} \rightarrow \infty$. As a result, we need to choose $\widetilde{R}(\zeta)$ satisfy that

$$
-\max _{0<\zeta<t_{f}}\left\{\frac{A^{2}(\widetilde{R}(\zeta))-f(\widetilde{R}(\zeta)) A(\widetilde{R}(\zeta))}{\partial A(\widetilde{R}(\zeta)) / \partial R}\right\}<\widetilde{R}^{\prime}(\zeta)<0,
$$

where $F=\left(x_{f}, t_{f}\right)$. With (63), the Goursat problem has a unique global $C^{1}$ solution in $\Omega_{8}$.

Similarly, in $\Omega_{6}$, we prescribe data as follows:

$$
\begin{aligned}
& \bar{R}(t)=R_{3}\left(\bar{x}_{1}(t), t\right), \quad \text { on } \bar{x}_{1}(t), \\
& \bar{\theta}(t)=\theta_{2}\left(\bar{x}_{2}(t), t\right), \quad \text { on } \bar{x}_{2}(t) .
\end{aligned}
$$

For $\forall(x, t) \in \Omega_{6}$, a characteristic passing by $(x, t)$ can be defined by

$$
\frac{d \widetilde{t}_{2}(x)}{d x}=\frac{1}{A\left(R\left(x, \widetilde{t}_{2}(x)\right)\right)}, \quad \widetilde{t}_{2}\left(\bar{x}_{1}(v)\right)=v,
$$

where $\left(\bar{x}_{1}(v), v\right)$ is the intersection point of this characteristic and the characteristic $\bar{x}_{1}(t)$. Along the characteristic $\tilde{t}_{2}(x)$, one has $d R / d x=0$, that is, $R=\bar{R}(v)$. Clearly, the $C^{0}$ norm of $R$ is bounded. In order to estimate $R_{t}$, we need to discuss $d \widetilde{t}_{2}(x) / d v$. From (65), one has 
$\tilde{t}_{2}(x)=v+(1 / A(R))\left(x-\bar{x}_{1}(v)\right)$. Then, $\tilde{t}_{2}(x) / d v=1-$ $(f(R) / A(R))-\left(x-\bar{x}_{1}(v)\right)(\partial A / \partial R)(d R / d v) / A^{2}(R)$. According to Remark 6, there at least exists a point $\left(x\left(v_{1}\right), v_{1}\right)$ such that $R_{x}\left(v_{1}\right)<0$. Then, $d R /\left.d v\right|_{v=v_{1}}=R_{t}+\left.R_{x} f(R)\right|_{v=v_{1}}=(f(R)-$ $A(T)) R_{x}\left(v_{1}\right)>0$. Note that $x-\bar{x}_{1}(v)>0$, there exists $x$ such that $R_{t} \rightarrow \infty$. As a result, we need to choose $\bar{R}(v)$ satisfy that

$$
0<\bar{R}^{\prime}(v)<\min _{t_{n}<v<T}\left\{\frac{A^{2}(\bar{R}(v))-f(\bar{R}(v)) A(\bar{R}(v))}{\partial A(\bar{R}(v)) / \partial R}\right\},
$$

where $N=\left(x_{n}, t_{n}\right)$. With (66), the Goursat problem has a unique global $C^{1}$ solution in $\Omega_{6}$.

In addition, with the same analysis, the Goursat problem always has a unique global $C^{1}$ solution in $\Omega_{5}, \Omega_{7}$. Therefore, under the conditions (63) and (66), we have got $C^{1}$ solutions in the domains $\Omega_{5}, \Omega_{6}, \Omega_{7}, \Omega_{8}$ and the solutions can be defined

$$
(R(x, t), \theta(x, t))= \begin{cases}\left(R_{5}(x, t), \theta_{5}(x, t)\right), & \text { for }(x, t) \in \Omega_{5}, \\ \left(R_{6}(x, t), \theta_{6}(x, t)\right), & \text { for }(x, t) \in \Omega_{6}, \\ \left(R_{7}(x, t), \theta_{7}(x, t)\right), & \text { for }(x, t) \in \Omega_{7}, \\ \left(R_{8}(x, t), \theta_{8}(x, t)\right), & \text { for }(x, t) \in \Omega_{8} .\end{cases}
$$

We have constructed solutions in the domain $D_{T}$. Let $h_{1}(t)=$ $R(0, t)-g_{1}(\theta(0, t), t)$, and $h_{2}(t)=\theta(1, t)-g_{2}(R(1, t), t)$; then there exist $h_{1}, h_{2} \in C^{1}([0, T])$ such that systems (10) (12) have a $C^{1}$ solution $(R(x, t), \theta(x, t))$ on the domain $D_{T}$ satisfying $R(x, 0)=R_{0}(x), \theta(x, 0)=\theta_{0}(x), R(x, T)=R_{T}(x), \theta(x, T)=$ $\theta_{T}(x)$, for $0 \leq x \leq 1$. The proof is completed.

Remark 17. In [10], an optimal control model of distributed parameter systems (DPSs) is presented to discuss the polymer injection strategies. Compared with [10], the differences of our paper are (1) the considered model is a hyperbolic system and the maximum principle does not hold here; (2) the desired outputs can be achieved by controlling the boundary inputs.

\section{An Example}

In this section, an example is presented to demonstrate the effectiveness of our results.

Example 18. For system (10), we define that

$$
\begin{array}{ll}
R(x, 0)=R_{0}(x)=C+\varepsilon \phi_{1}(x), & \phi_{1}^{\prime}(x)>0, \\
R(x, T)=R_{T}(x)=C+\varepsilon \phi_{2}(x), & \phi_{2}^{\prime}(x)<0,
\end{array}
$$

where $C>0$ is a constant, which denotes the equilibrium of the initial and terminal states. $m_{1} \leq \phi_{1}(x) \leq M_{1}, m_{2} \leq$ $\phi_{2}(x) \leq M_{2}$, and $m_{1}, M_{1}, m_{2}, M_{2}$ are all constants. $\varepsilon>0$ is also a constant, which will be chosen in the following.

As a result, one has $C_{1} \leq R(x, t) \leq C_{2}$ with $C_{1}=C+$ $\varepsilon \min \left\{m_{1}, m_{2}\right\}$ and $C_{2}=C+\varepsilon \max \left\{M_{1}, M_{2}\right\}$. Let $f(R)=R-$ $C_{2}-\eta, A(R)=f^{\prime}(R) R+f(R)=2 R-C_{2}-\eta$, and $\eta$ depends on $\varepsilon$ and is also decided later. In the following, we will choose appropriate $\varepsilon$ to satisfy conditions (57), (58), (63), and (66).

According to Theorem 12 , one has $\widetilde{R}_{0}^{\prime}\left(t_{p}\right)=(k-$ $\left.A\left(R_{0}(0)\right)\right)(\partial R / \partial x)=\left(k-A\left(R_{0}(0)\right)\right)\left(\partial R_{0} / \partial t\right) /(\partial x / \partial t)=(k-$ $\left.A\left(R_{0}(0)\right)\right)\left(\varepsilon \phi_{1}^{\prime}(x) / A\left(R_{0}(0)\right)\right)$. Note that $\widetilde{R}_{0}^{\prime}\left(t_{p}\right)<0$, an appropriate $\varepsilon$ can be chosen to satisfy condition (58). Applying the same method, it is also easy to choose an appropriate $\varepsilon$ to satisfy condition (57).

Let $\left(x_{1}\left(\xi_{1}\right), \xi_{1}\right)$ be the intersection point of the characteristic (65) and the straight line $P Q$. Because the straight line $P Q$ is constructed, we can choose that $\left|\widetilde{R}_{0}^{\prime}\left(\xi_{1}\right)\right|<$ $\min \left\{\left|\widetilde{R}_{0}^{\prime}\left(t_{p}\right)\right|,\left|\widetilde{R}_{0}^{\prime}\left(t_{q}\right)\right|\right\}$. As a result, (66) will be satisfied if condition (57) is satisfied.

Similarly, let $\left(x_{1}\left(v_{1}\right), v_{1}\right)$ be the intersection point of the characteristic (62) and the straight line $P Q$. Because the straight line $P Q$ is constructed, we can choose that $\left|\widetilde{R}_{0}^{\prime}\left(\nu_{1}\right)\right|<$ $\min \left\{\left|\widetilde{R}_{0}^{\prime}\left(t_{p}\right)\right|,\left|\widetilde{R}_{0}^{\prime}\left(t_{q}\right)\right|\right\}$. As a result, (63) will be satisfied if condition (58) is satisfied. So, there exists a positive constant $\varepsilon$ to satisfy conditions (57), (58), (63), and (66). In addition, for the chosen $\varepsilon$, it is easy to find out a constant $\eta(0<\eta<C-3 \varepsilon)$ to satisfy $f(R)=R-C_{2}-\eta<0$ and $A(R)=f^{\prime}(R) R+f(R)=$ $2 R-C_{2}-\eta>0$. Therefore, all the conditions in Theorem 12 can be satisfied by using the constructed method. That is, the conclusion of Theorem 12 is valid.

Remark 19. Compared with [20,21], the difference in this paper is the fact that one cannot avoid the phenomenon of blowup. Hence, one important goal for EORs is to find the controllers $h_{1}(t)$ and $h_{2}(t)$ to make sure that the blowup points of EORs are beyond the domain we fixed. As a result, there exist more complications in the controlling process as described in Section 3. The example we give here is to illustrate that the controllers are achievable and it can be applied in real problems.

\section{Conclusions}

In this paper, we have discussed the exact boundary controllability of a class of enhanced oil recovery models. By using a constructed method, it has been shown that the enhanced oil recovery systems with nonlinear boundary conditions is exactly boundary controllable. Moreover, an interval of the control time has also been presented to be optimal. Finally, an example has been provided to illustrate the effectiveness of the obtained criterion.

\section{Conflict of Interests}

The authors declare that there is no conflict of interests regarding the publication of this paper.

\section{Acknowledgments}

This work was jointly supported by the National Natural Science Foundation of China under Grant no. 61203146, the Educational Commission of Sichuan province under Grant no. 12ZA198, the China Postdoctoral Fund under Grant no. 2013M541589, the Jiangsu Postdoctoral Fund under Grant 
no. 1301025B, the Open Fund of State Key Laboratory of Oil and Gas Reservoir Geology and Exploitation under Grant no. PLN1130, and the University Research Funds under Grants nos. 2012XJZ029, 2012XJZT005, and 2013XJZT004.

\section{References}

[1] L. M. Abriola, T. J. Dekker, and K. D. Pennell, "Surfactantenhanced solubilization of residual dodecane in soil columns2. Mathematical modeling," Environmental Science and Technology, vol. 27, no. 12, pp. 2341-2351, 1993.

[2] P. Daripa and G. Paşa, "An optimal viscosity profile in enhanced oil recovery by polymer flooding," International Journal of Engineering Science, vol. 42, no. 19-20, pp. 2029-2039, 2004.

[3] B. Ju, T. Fan, and M. Ma, "Enhanced oil recovery by flooding with hydrophilic nanoparticles," China Particuology, vol. 4, no. 1, pp. 41-46, 2006.

[4] K. C. Taylor and H. A. Nasr-El-Din, "Water-soluble hydrophobically associating polymers for improved oil recovery: a literature review," Journal of Petroleum Science and Engineering, vol. 19, no. 3-4, pp. 265-280, 1998.

[5] J. Hou, Z. Liu, S. Zhang, X. Yue, and J. Yang, "The role of viscoelasticity of alkali/surfactant/polymer solutions in enhanced oil recovery," Journal of Petroleum Science and Engineering, vol. 47, no. 3-4, pp. 219-235, 2005.

[6] D. A. Z. Wever, F. Picchioni, and A. A. Broekhuis, "Polymers for enhanced oil recovery: a paradigm for structure-property relationship in aqueous solution," Progress in Polymer Science, vol. 36, no. 11, pp. 1558-1628, 2011.

[7] Q. Wang, X. Fang, B. Bai et al., "Engineering bacteria for production of rhamnolipid as an agent for enhanced oil recovery," Biotechnology and Bioengineering, vol. 98, no. 4, pp. 842-853, 2007.

[8] R. Sen, "Biotechnology in petroleum recovery: the microbial EOR," Progress in Energy and Combustion Science, vol. 34, no. 6, pp. 714-724, 2008.

[9] S. M. Desouky, M. M. Abdel-Daim, M. H. Sayyouh, and A. S. Dahab, "Modelling and laboratory investigation of microbial enhanced oil recovery," Journal of Petroleum Science and Engineering, vol. 15, no. 2-4, pp. 309-320, 1996.

[10] Y. Lei, S. Li, X. Zhang, Q. Zhang, and L. Guo, "Optimal control of polymer flooding based on maximum principle," Journal of Applied Mathematics, vol. 2012, Article ID 987975, 20 pages, 2012.

[11] N. Lai, X. Qin, Z. Ye, Q. Peng, Y. Zhang, and Z. Ming, "Synthesis and evaluation of a water-soluble hyperbranched polymer as enhanced oil recovery chemical," Journal of Chemistry, vol. 2013, Article ID 824785, 11 pages, 2013.

[12] E. L. Isaacson and J. B. Temple, "Analysis of a singular hyperbolic system of conservation laws," Journal of Differential Equations, vol. 65, no. 2, pp. 250-268, 1986.

[13] D. Schaeffer and M. Shearer, "Riemann problems for nonstrictly hyperbolic $2 \times 2$ systems of conservation laws," Transactions of the American Mathematical Society, vol. 304, pp. 267-305, 1987.

[14] A. Tveito and R. Winther, "Existence, uniqueness, and continuous dependence for a system of hyperbolic conservation laws modeling polymer flooding," SIAM Journal on Mathematical Analysis, vol. 22, no. 4, pp. 905-933, 1991.

[15] A. J. de Souza, "Wave structure for a nonstrictly hyperbolic system of three conservation laws," Mathematical and Computer Modelling, vol. 22, no. 9, pp. 1-29, 1995.
[16] P. Daripa, J. Glimm, B. Lindquist, and O. McBryan, "Polymer floods: a case study of nonlinear wave analysis and of instability control in tertiary oil recovery," SIAM Journal on Applied Mathematics, vol. 48, no. 2, pp. 353-373, 1988.

[17] J. Lu, D. W. C. Ho, and Z. Wang, "Pinning stabilization of linearly coupled stochastic neural networks via minimum number of controllers," IEEE Transactions on Neural Networks, vol. 20, no. 10, pp. 1617-1629, 2009.

[18] J. Lu, J. Kurths, J. Cao, N. Mahdavi, and C. Huang, "Synchronization control for nonlinear stochastic dynamical networks: pinning impulsive strategy," IEEE Transactions on Neural Networks and Learning Systems, vol. 23, no. 2, pp. 285-292, 2012.

[19] W. Yu, G. Chen, and J. Lü, "On pinning synchronization of complex dynamical networks," Automatica, vol. 45, no. 2, pp. 429-435, 2009.

[20] T. Li and B.-Y. Zhang, "Global exact controllability of a class of quasilinear hyperbolic systems," Journal of Mathematical Analysis and Applications, vol. 225, no. 1, pp. 289-311, 1998.

[21] D.-X. Kong and H. Yao, "Global exact boundary controllability of a class of quasilinear hyperbolic systems of conservation laws. II," SIAM Journal on Control and Optimization, vol. 44, no. 1, pp. 140-158, 2005.

[22] T. Li, Global Classical Solutions for Quasilinear Hyperbolic Systems, Wiley, 1994. 


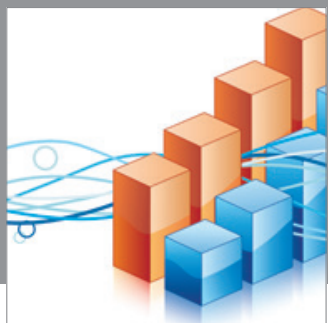

Advances in

Operations Research

mansans

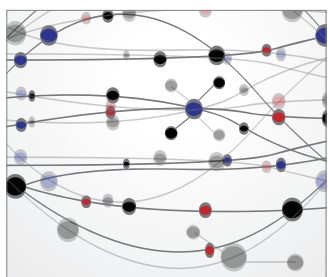

The Scientific World Journal
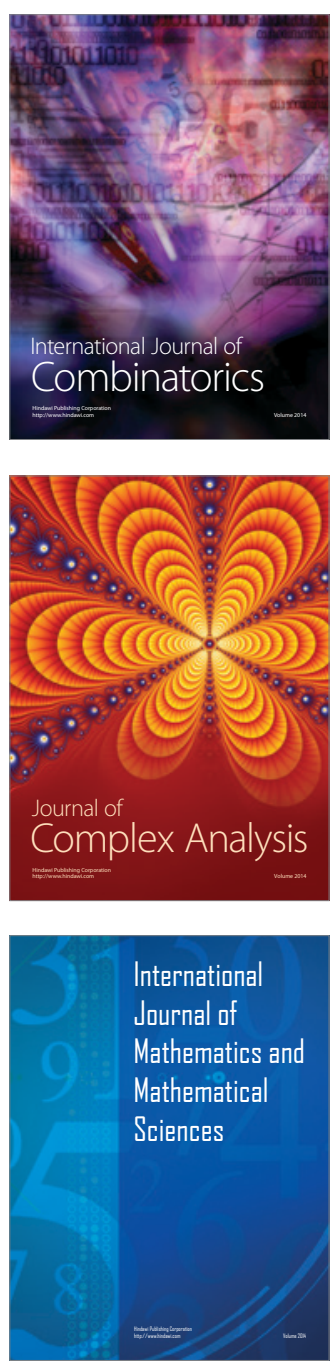
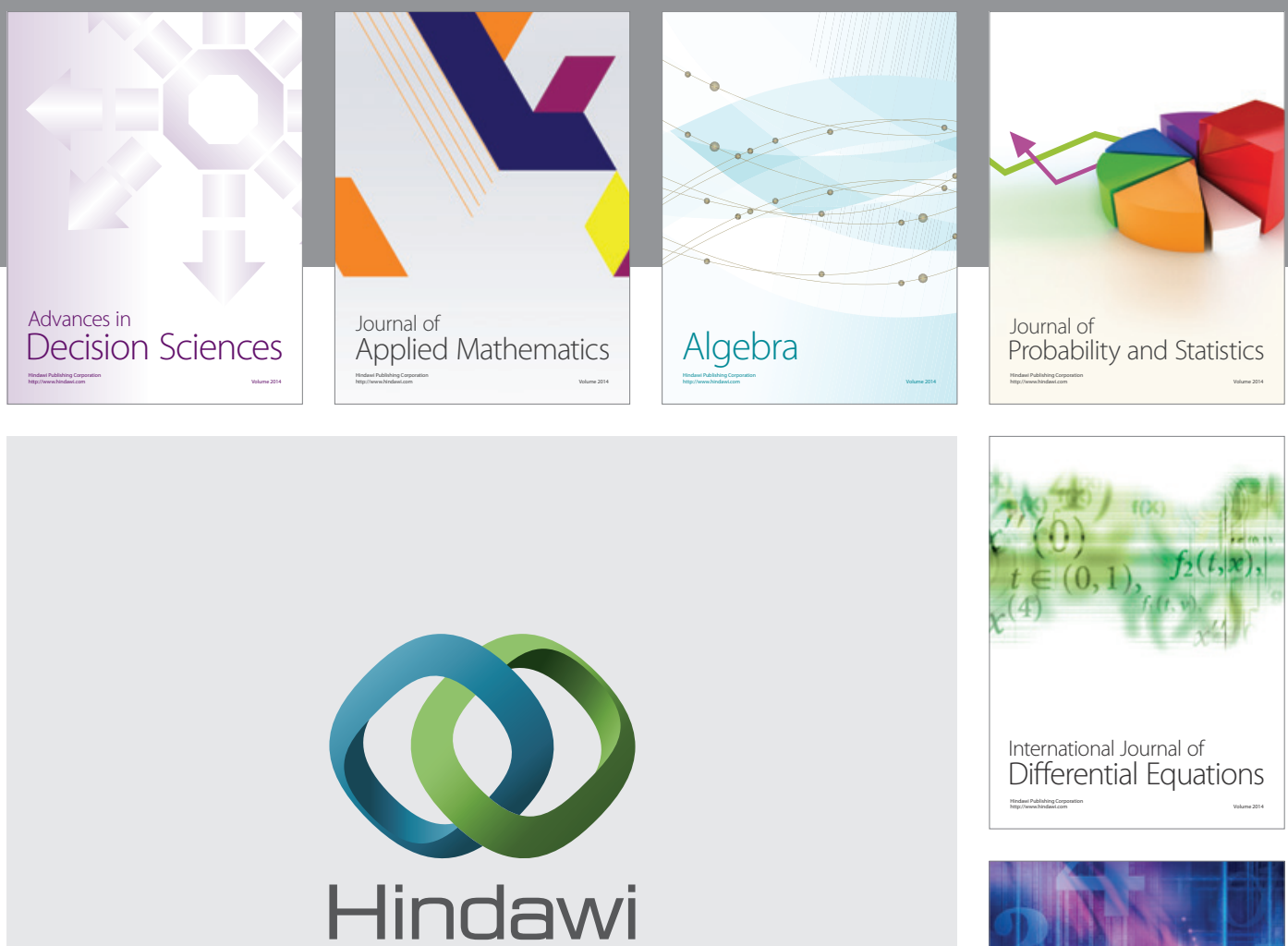

Submit your manuscripts at http://www.hindawi.com
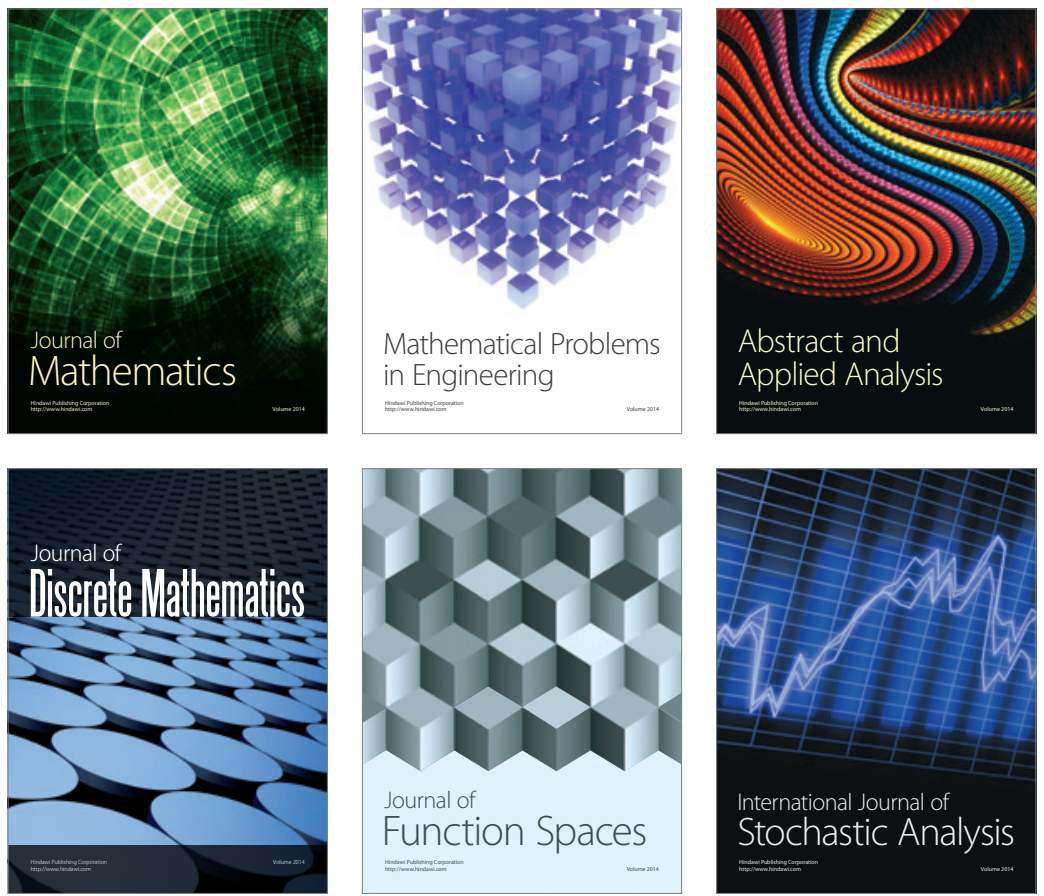

Journal of

Function Spaces

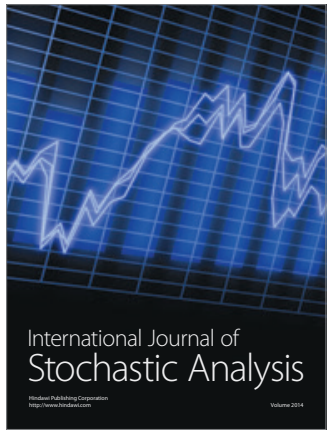

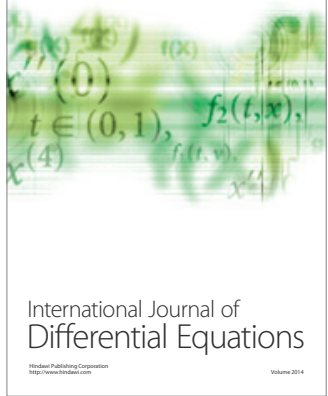
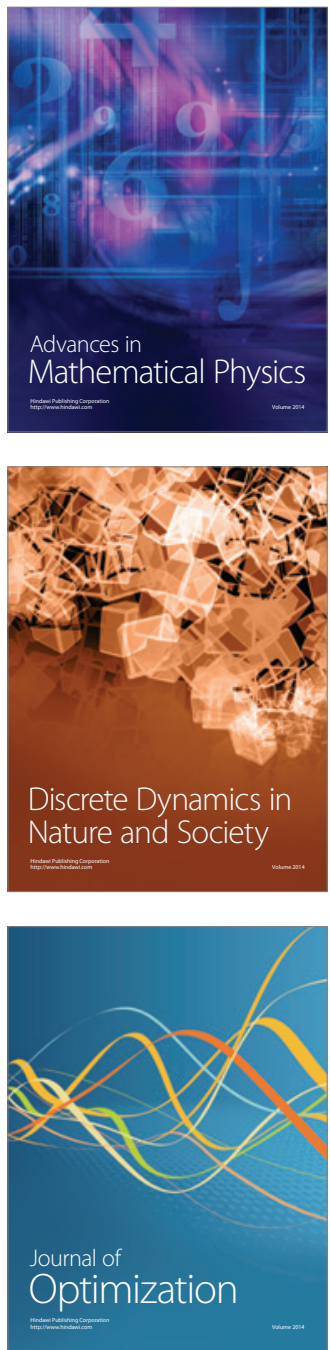